\title{
El pueblo de San Jerónimo en el Cusco: \\ Ayllus y organización territorial durante el siglo XIX
}

\author{
The town of San Jeronimo in Cusco: Ayllus and territorial \\ organization during the nineteenth century \\ Recibido: junio 23 de 2016 | Revisado: julio 20 de 2016 | Aceptado: setiembre 15 de 2016
}

DAvid CeRPa Espinosa ${ }^{1}$

1 Universidad de San Martín de Porres - Perú dcerpae@usmp.pe

\begin{abstract}
Resumen
El pueblo de San Jerónimo ubicado muy cerca de la ciudad del Cusco tiene una historia vinculada con las antiguas familias reales incaicas. Durante los tiempos coloniales hasta los últimos ańos del siglo XIX, en este pueblo, se encontraban reducidos algunos de los más importantes ayllus del Cusco. El propósito de esta invesigación es establecer un paralelo entre la organización de los ayllus de San Jerónimo, durante tres momentos específicos del siglo XIX y su probable localización espacial, a partir de la lectura de algunos mapas de fines del siglo XX y una imagen satelital actual.
\end{abstract}

Palabras clave: ayllu, hacienda, organización social, estructura espacial

\begin{abstract}
The town of San Jerónimo is located near the city of Cusco, and its history is related with the ancient royal Inca families. During the colonial period up to the last years of the 19th century, some of the most important ayllus of Cusco were located in this town. The purpose of this research is to establish a parallel between the organization of the ayllus of San Jerónimo during three specific moments of the 19th century and its probable spatial location, based on the revision of maps from the late 20th Century and current satellite images.
\end{abstract}

Keywords: Ayllu, farm, social organization, spatial structure 


\section{Introducción}

El pueblo de San Jerónimo está ubicado a unos $10 \mathrm{Km}$. al este del centro histórico del Cusco, dentro de la cuenca del río Huatanay, importante afluente del río Vilcanota, y lugar donde se desarrollaron los asentamientos directamente vinculados con la antigua capital del imperio de los Incas.

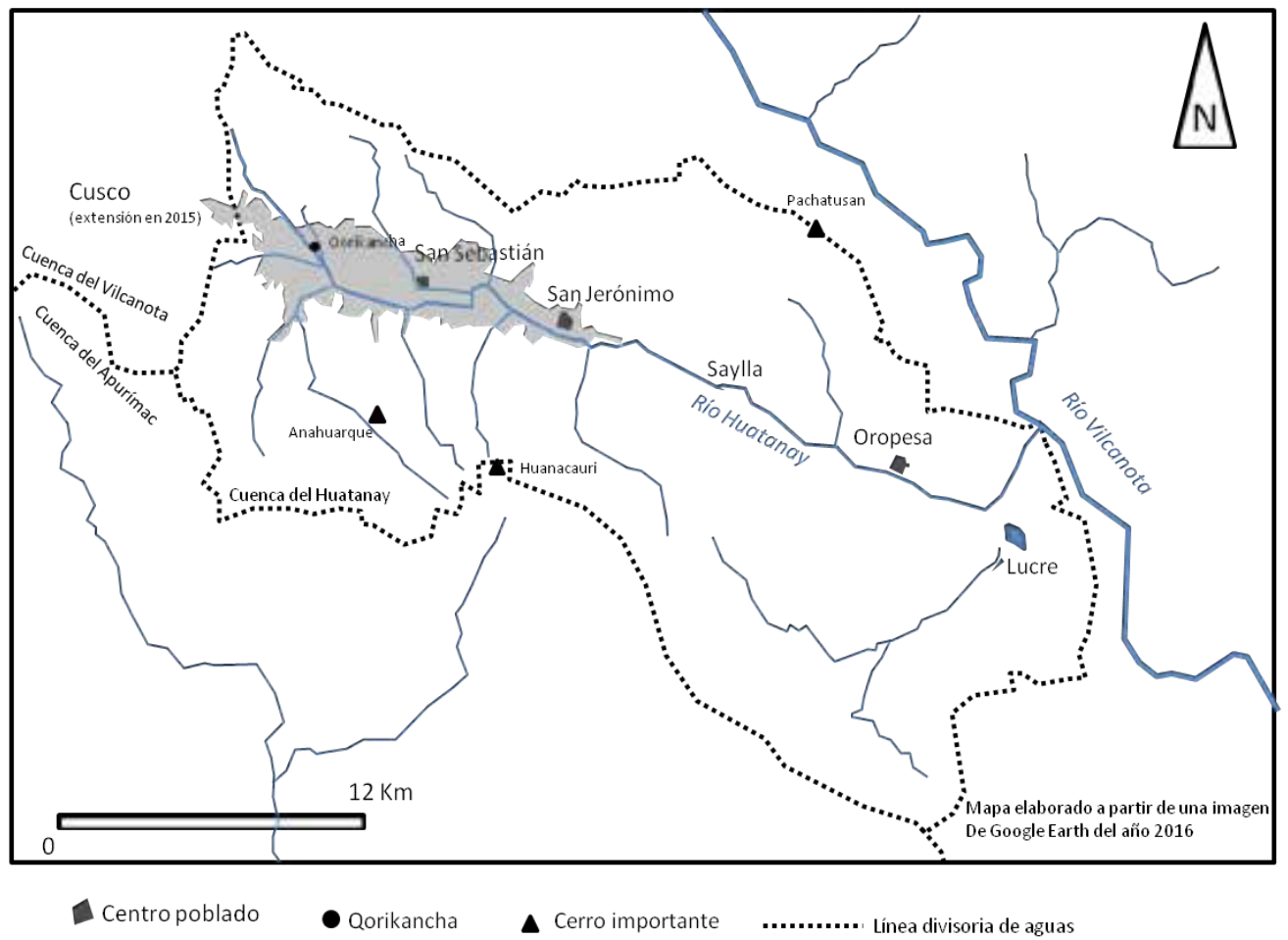

Figura 1. Localización del pueblo de San Jerónimo en relación con la cuenca del río Huatanay y la ciudad del Cusco en 2016

Durante el siglo XIX, la ciudad del Cusco estaba organizada en nueve repartimientos (Archivo Regional del Cusco, Matrículas de Indígenas de 1836) : Belén, Santiago, Hospital (San Pedro), Santa Ana, San Cristóbal, San Blas, San Sebastián, San Jerónimo y la Matriz, estando San Sebastián y San Jerónimo fuera del área urbana. Actualmente, la ciudad del Cusco ha crecido de tal forma que San Sebastián es ya parte de esta, mientras que San Jerónimo está casi rodeado por áreas urbanizadas (Figura 1). Es importante mencionar que el concepto de repartimiento se utilizó tanto en tiempos virreinales como durante la primera mitad del siglo XIX para designar un sector territorial que comprendía uno o varios pueblos, y que, a partir de la segunda mitad de dicho siglo, fue reemplazado por lo que conocemos actualmente como provincias y distritos.
El siglo XIX marcó el final del Virreinato del Perú y el inicio del período republicano, por lo tanto, fue un tiempo de muchos cambios en diversos aspectos, entre ellos el social y el territorial. Resulta extremadamente complejo tratar todos estos cambios en relación con el conjunto de repartimientos de la ciudad del Cusco; sin embargo, trataremos de establecer una visión preliminar de lo que sucedió en el repartimiento de San Jerónimo durante este período, enfatizando el estudio de los ayllus que formaban parte de este en su dimensión espacial y territorial.

En tal sentido, es importante centrarse en el siglo XIX debido a que durante la primera mitad de esa época, se mantuvieron en pie muchas de las estructuras organizacionales correspondientes al período virreinal y que se fueron modificando, paulatinamente, a 
partir de la segunda mitad de dicho siglo; es interesante notar que dichas transformaciones se consolidaron entrado ya el siglo XX. Sin embargo, somos conscientes que muchas de estas estructuras están aún vigentes en varios aspectos de la vida social y territorial del Cusco y de muchos lugares de los Andes, y que, a pesar del tiempo transcurrido es innegable que su origen más lejano está necesariamente vinculado con los tiempos prehispánicos.

Efectivamente, una de las principales instituciones que prevaleció desde la época prehispánica fueron los ayllus, los mismos que sufrieron importantes transformaciones durante el período virreinal, siendo su conocimiento aún difícil de abordar debido a los vacíos documentales y las contradicciones conceptuales que dificultan su estudio. Sin embargo, el siglo XIX representa un período en el cual se realizaron importantes registros, cuyo estudio podría contribuir con un mejor conocimiento del carácter de los ayllus y su organización tanto a nivel social como territorial.

Una de las principales fuentes documentales que permite evaluar la evolución de los ayllus durante la primera mitad del siglo XIX - no solo en el Cusco, sino también en otros lugares del Perú - lo constituyen los libros de Matrículas de Contribuciones que se conservan, actualmente, tanto en el Archivo General de la Nación como en los Archivos Regionales del Perú, entre ellos, el Archivo Regional del Cusco.

Dichos libros se elaboraron durante el período comprendido entre 1826 y 185 , aproximadamente, y fueron realizados con el objetivo de recaudar fondos para la reconstrucción del Perú luego de la guerra de la Independencia, tal como lo sostiene Basadre (2005) [1939]. Desafortunadamente, no todos los libros de contribuciones se han conservado, por lo tanto, existe un importante desbalance en cuanto a la disponibilidad de la información a nivel nacional. Para el caso del departamen- to del Cusco, podemos encontrar abundante información a nivel provincial; sin embargo, para el caso del Cercado hay notables vacíos debido a que es probable que algunos de estos libros se hayan perdido. Sin embargo, a pesar de esto, los documentos que se han conservado son de gran valor para comprender muchos aspectos relacionados con la temática que queremos abordar.

No se puede decir que exista una clasificación estricta de estos documentos; sin embargo, podríamos organizar estos en dos grandes grupos: el primer grupo, conformado por los libros de matrículas de contribución predial, estaban clasificados en dos subgrupos: los libros de contribuciones de predios urbanos y los libros de contribuciones de predios rústicos. Los primeros se organizaban espacialmente por calles, en función a la ubicación del predio respectivo dentro del área urbana, mientras que el segundo se organizaba por haciendas, ubicadas estas en las afueras de la ciudad. En este subgrupo, podemos encontrar también los libros de contribución industrial.

El segundo grupo, lo constituían los libros de matrículas de contribución personal, en los cuales había también dos subgrupos: los libros de patentes (Archivo General de la Nación 1839) y los libros de matrículas de indígenas. El primer subgrupo estaba comprendido por los gremios de profesionales $\mathrm{y}$ artesanos, y el segundo subgrupo estaba comprendido por las matrículas de indígenas, las mismas que se organizaban por repartimientos, habiendo en cada uno de estos un número determinado de ayllus, estando dedicados los integrantes de los ocho primeros repartimientos a la actividad agrícola, mientras que en el Noveno Repartimiento, conocido también como Matriz (Archivo Regional del Cusco Matrículas de Indígenas 1836), había un ayllu, llamado Yanaconas y el resto estaba conformado por gremios de artesanos al igual que en el caso de los libros de patentes. Es probable que la diferencia entre un artesano inscrito en el registro de 
patentes en relación con el artesano registrado en la matrícula de indígenas haya estado en relación con el origen del mismo. En el caso de los centros poblados no comprendidos por el cercado, había un grupo de contribuyentes no indígenas que se les conocía también como Castas.

A grandes rasgos, se puede decir que el primer grupo está vinculado con la organización espacial del Cusco durante la primera mitad del siglo XIX, mientras que el segundo grupo está vinculado con la organización social del Cusco durante dicho período. Sin embargo, del total de cuatro subgrupos el más difícil de localizar territorialmente es el último, debido a que, como se puede observar, el primer y tercer subgrupos están claramente vinculados con el espacio urbano; el segundo puede localizarse, con cierta precisión, dentro del área no urbana gracias a la cartografía disponible tanto del siglo XIX como la del siglo XX; el cuarto subgrupo resulta difícil de localizar debido a que se cuenta con muy pocas referencias cartográficas que permitan determinar la ubicación exacta de los ayllus tanto en el ámbito rural como en el urbano.

\section{Planteamiento del problema}

Dado que los libros de matrículas de indígenas registran los ayllus por repartimiento, es lógico pensar que estos ayllus debieron tener una ubicación territorial específica dentro de cada uno de estos, tal como lo tuvieron los predios urbanos y rústicos. Por otro lado, a pesar de que los libros de patentes no especifican la ubicación territorial de los gremios de artesanos y profesionales, es muy probable que estos se hayan concentrado en el barrio central de la ciudad (Matriz). En tal sentido, el caso de los ayllus plantea aún un problema teórico debido a que, a pesar de que los documentos especifican que los contribuyentes indígenas estaban vinculados con el trabajo en las tierras agrícolas, no se especifica nada sobre cuál fue su lugar de residencia dentro de cada repartimiento. Este vacío es, en sí mismo, un problema que aún no se ha podido resolver para el caso no solo de la ciudad del Cusco, sino también para el de muchos centros poblados del área andina. El presente artículo no pretende plantear una discusión teórica sobre el tema ni mucho menos resolver el problema; sin embargo, el objetivo del mismo es presentar algunas hipótesis de cómo pudieron definirse los ayllus como entidades territoriales en el repartimiento de San Jerónimo.

Para poder plantear estas hipótesis, haremos una breve revisión de algunos datos concernientes a tres momentos específicos del siglo XIX: los años 1836, 1876 y 1888. Antes de realizar esta revisión analizaremos, brevemente, algunos elementos que conciernen al origen de los ayllus relacionados con el cercado del Cusco.

\section{Antecedentes históricos}

Antes de abordar el análisis de los documentos correspondientes al siglo XIX, es interesante revisar dos documentos coloniales que hacen referencia a ayllus cuyos nombres van a coincidir con los nombres de algunos ayllus que pertenecían a los repartimientos del Cusco. Me refiero básicamente a la $R e-$ lación de las Fábulas y Ritos de los Incas de Cristóbal de Molina (2008) y la Relación de las Guacas del Cuzco que Bernabé Cobo escribiera en 1653 (1981), como parte de su Historia del Nuevo Mundo.

En el primer documento, el cronista Cristóbal de Molina hace una extensa explicación de diversos mitos y festividades, de las que destacamos una en particular, que corresponde a la fiesta de la Citua, la cual tenía lugar en el mes de agosto, mes conocido como Coya Raymi. Durante esta fiesta, unos personajes salían del Cusco hacia los cuatro Suyos con la finalidad de purificar la ciudad, dirigiéndose hacia lugares alejados. No explicaremos mayores detalles de esta fiesta; sin embargo, es interesante extraer de aquí una 
información que permite dar fundamento al origen Inca de algunos de los ayllus del Cusco. En tal sentido, de la crónica de Cristóbal de Molina (2008) extraemos lo siguiente:

Eran estos indios que llevaban estas voces hacia Collasuyo, los que salían del Cuzco, de la generación de Uscamayta Ayllu, Apo Mayta Ayllu, Hahuaynin Ayllu, Sutic y Maras Ayllu, Cuycussa Ayllu.

(...) Los que salían hacia el poniente que es a Chinchaysuyo salían dando las mismas voces y estos eran de la generación Capac Ayllu y Hatun Ayllu y Vicaquirao y Chahuin Cuzco Ayllo, y Arayraca Ayllu y otros de Uro.

(...) Los que llevaban las voces de la parte de Antisuyo eran de las generaciones siguientes: Sucsu Panaca Ayllu, Aucaylli Ayllu, Tarpuntay Ayllu, Sano Ayllu.

(...) Los que iban a la parte de Contisuyo eran de las generaciones siguientes: Raura Panaca Ayllu y Chima Panaca Ayllu y Masca Panaca Ayllu y Quesco Ayllu.

Es necesario indicar que el texto de Moli- na ha sido actualizado por el Dr. Julio Calvo Pérez, considerando, además, que la edición de 2008 contiene también la versión paleográfica del mismo. Las palabras, en letra cursiva, son tal como aparecen en el texto de la versión actualizada.

El otro documento, vale decir, la Relación de las Guacas del Cuzco, escrita por el padre Bernabé Cobo, y que ha sido transcrita y estudiada por John Rowe (1981), hace referencia a las líneas llamadas Ceques, que partían desde el Qorikancha en varias direcciones, conteniendo cada una de estas líneas un número variable de huacas o adoratorios. Según este documento, algunas de estas líneas estaban a cargo de determinados ayllus, de los cuales algunos de ellos fueron aparentemente reducidos en los repartimientos del cercado del Cusco. Tom Zuidema realizó importantes estudios sobre este sistema, fundamentalmente, en su obra El Sistema de Ceques del Cuzco, La organización Social de la Capital de los Incas (1995) [1964], sin embargo, cabe destacar también el trabajo de John Rowe, Brian Bauer (2000) [1998], entre otros. A continuación, se muestra un diagrama esquemático de los ceques.

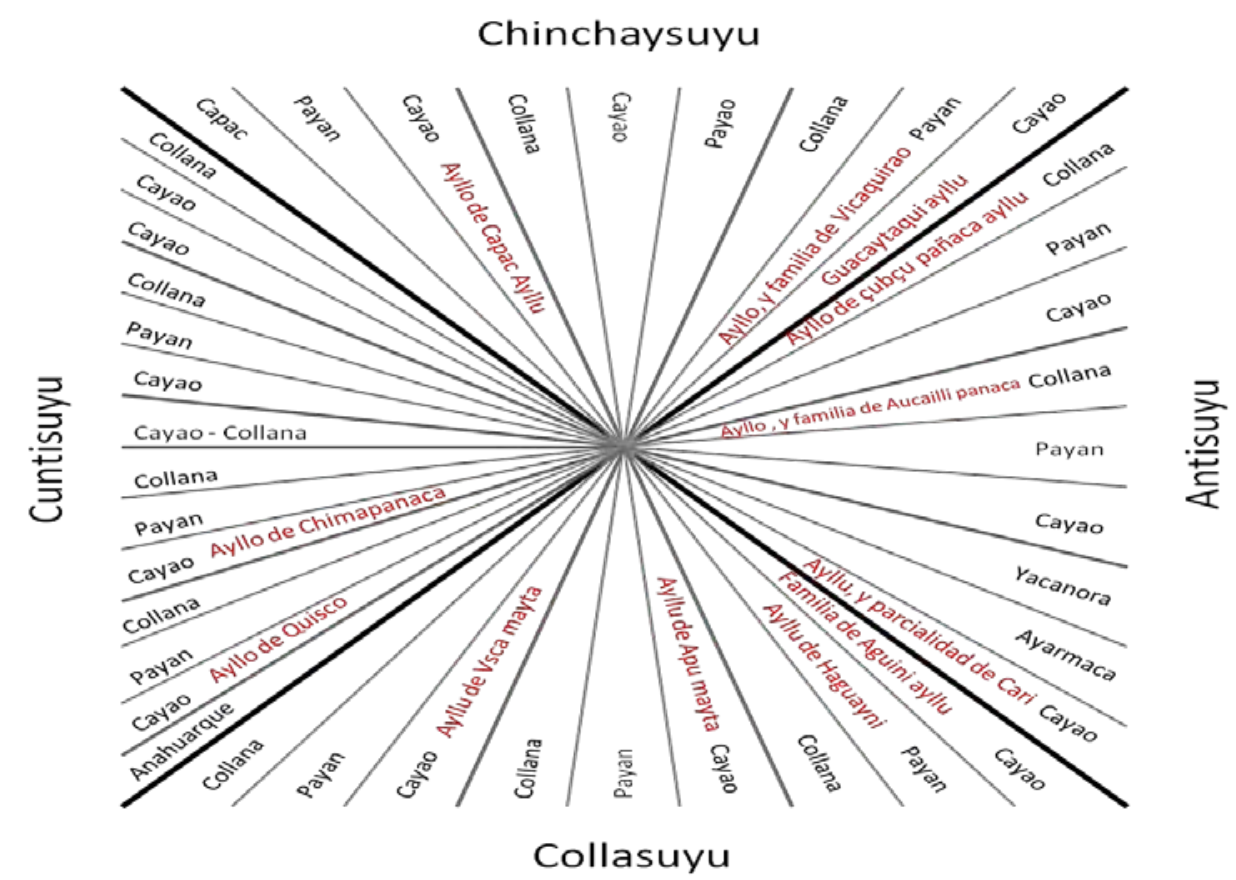

Figura 2. Diagrama esquemático de los Ceques del Cusco. Interpretación gráfica de la relación de Bernabé Cobo. 
El diagrama mostrado en la Figura 2, es una interpretación esquemática del documento del padre Bernabé Cobo. No es de ningún modo una reconstrucción de las direcciones de los ceques, sino que simplemente se busca aquí mostrar, de manera sintéti$\mathrm{ca}$, los ceques agrupados en función de los cuatro Suyos. Como puede verse, se trataba de un total de 41 direcciones organizadas, en cuatro partes, de acuerdo con los cuatro suyos: Nueve desde el Chinchaysuyu; nueve desde el Antisuyu; nueve desde el Collasuyu y catorce desde el Cuntisuyu. Téngase en cuenta que este diagrama muestra a los Ceques más como franjas que como líneas, a fin de facilitar su lectura.

Como puede verse también, cada Ceque tenía, en la mayoría de los casos, uno de los nombres siguientes: Collana, Payan y Cayao, habiendo también otros ceques cuyos nombres eran Cápac, Yacanora, Ayarmaca y Anahuarque. Sin embargo, en la mayoría de los casos, los ceques formaban grupos de tres, siguiendo el orden correlativo Collana-Payan-Cayao, aunque en algunos casos dicho orden variaba, tal vez por un error de transcripción. Asimismo, algunos de estos ceques estaban a cargo de un ayllu específico, tal como se indica en color rojo en dicho diagrama.

Al comparar el diagrama con las direcciones que seguían los que participaban en la festividad de la Citua, observamos que aquellos ayllus que figuran en la relación de ceques de Cobo aparecen también en el documento de Molina:

\section{Tabla 1}

Comparación entre los ayllus relacionados con las direcciones de la Citua, los ayllus del sistema de ceques y los linajes reales descritos por Sarmiento de Gamboa.

\begin{tabular}{|c|c|c|c|}
\hline & $\begin{array}{l}\text { Linajes incas según Sarmiento } \\
\text { de Gamboa }\end{array}$ & $\begin{array}{l}\text { Direcciones de la Citua } \\
\text { (Cristóbal de Molina) }\end{array}$ & $\begin{array}{l}\text { Sistema de ceques } \\
\text { (Bernabé Cobo) }\end{array}$ \\
\hline \multirow[t]{6}{*}{ Collasuyo } & Mayta Ccapac & Uscamayta Ayllu & Ayllu de Vsca mayta \\
\hline & Ccapac Yupanqui & Apo Mayta Ayllu & Ayllu de Apumayta \\
\hline & & Sutic y Maras Ayllu & \\
\hline & & Cuycussa Ayllu & \\
\hline & Lloqui Yupanqui & Hauaynin Ayllu & Ayllu de Haguayni \\
\hline & & & Familia de Aguini ayllu \\
\hline \multirow[t]{6}{*}{ Chinchaysuyo } & Tupac Inca Yupanqui & Capac Ayllu & Ayllu de Capac Ayllu \\
\hline & Pachacuti Inca Yupanqui & Hatun Ayllu & \\
\hline & Inca Rocca & Vicaquirao Ayllu & Ayllu y familia de Vicarquirao \\
\hline & Ayar Cachi & Chahuin Cuzco Ayllu & \\
\hline & Ayar Uchu & Arayraca Ayllu & Huacaytaqui Ayllu \\
\hline & & Uro & \\
\hline \multirow[t]{5}{*}{ Antisuyo } & Viracocha & Sucsu Panaca Ayllu & Ayllu de cubcu pañaca ayllu \\
\hline & Yahuar-Huaccac & Aucaylli Ayllu & $\begin{array}{l}\text { Ayllu y familia de Aucailli } \\
\text { panaca }\end{array}$ \\
\hline & & Tarpuntay Ayllu & \\
\hline & & Sano Ayllu & \\
\hline & & & Ayllu y parcialidad de Cari \\
\hline \multirow[t]{4}{*}{ Cuntisuyo } & Sinchi Rocca & Raura Panaca Ayllu & \\
\hline & Manco Ccapac & Chima Panaca Ayllu & \\
\hline & & Masca Panaca Ayllu & \\
\hline & & Quesco Ayllu & Ayllu de Quisco \\
\hline
\end{tabular}


Al observar la Tabla 1, vemos que existen coincidencias entre los dos grupos de ayllus, sobre todo en lo que concierne a su ubicación con respecto a los cuatro suyos. Sin embargo, la relación de Cristóbal de Molina muestra un mayor número de ayllus. Pedro Sarmiento de Gamboa (1988), cronista español del siglo XVI, vincula a un grupo de los ayllus con los gobernantes incas, mientras que otro grupo (en letra cursiva) corresponden a los diez ayllus que, según la leyenda, acompañaron a Manco Cápac hacia el Cusco. Sarmiento no nombra al ayllu Quesco, pero si nombra al ayllu Huacaytaqui, el cual no está en la lista de Molina.

\section{Los documentos de 1836}

Para el análisis de los ayllus de San Jerónimo, contamos con los datos que nos ofre-

Tabla 2

Ayllus de la parroquia de San Jerónimo en 1836

\begin{tabular}{|c|c|c|c|c|}
\hline Repartimiento & Ayllo & $\begin{array}{l}\text { Originarios } \\
\text { con tierras }\end{array}$ & $\begin{array}{l}\text { Forasteros } \\
\text { con tierras }\end{array}$ & $\begin{array}{l}\text { Forasteros } \\
\text { sin tierras }\end{array}$ \\
\hline $\begin{array}{l}\text { Octavo repartimiento } \\
\text { Parroquia de San } \\
\text { Jerónimo }\end{array}$ & $\begin{array}{l}\text { Ccollana } \\
\text { Chahuanccoscco } \\
\text { Ccallampa } \\
\text { Orccon } \\
\text { Uruhuaccama } \\
\text { Huiccahuiray } \\
\text { (Guiccahuiray) } \\
\text { Apumayta } \\
\text { Raurau } \\
\text { Anahuarqui y Chima } \\
\text { Andamachay } \\
\text { Sorama } \\
\text { Ccollanas } \\
\text { Estancia de } \\
\text { Runtubamba }\end{array}$ & $\begin{array}{c}10 \\
6 \\
12 \\
48 \\
17 \\
3 \\
7 \\
0 \\
183\end{array}$ & $\begin{array}{l}4 \\
5 \\
3 \\
7 \\
6 \\
0 \\
1\end{array}$ & $\begin{array}{c}9 \\
15 \\
28 \\
21 \\
32 \\
13 \\
5 \\
37 \\
265\end{array}$ \\
\hline
\end{tabular}

Lo primero que llama la atención al leer el listado de ayllus de San Jerónimo es la correspondencia de cinco de estos con los ay- cen, por un lado, las matrículas de predios rústicos, y por otro lado, las matrículas de indígenas del año 1836 (Archivo Regional del Cusco, matrículas de 1836). No aparece aquí el rubro de predios urbanos, debido a que San Jerónimo no era considerado como un centro poblado de tipo urbano, a pesar de que su trazo está definido por manzanas y calles.

Por otro lado, no aparecen aquí los libros de los gremios pues estos se agrupaban, tal como se explicó anteriormente, en el Noveno Repartimiento (matriz). La Tabla 2 nos muestra los ayllus de San Jerónimo en 1836 (tabla elaborada a partir de la matrícula de indígenas de dicho año que se encuentra en el Archivo Regional del Cusco).

llus que nombra Cristóbal de Molina, tales como Chahuanccoscco y Huiccahuiray (Vicaquirao) en el Chinchaysuyo; Apumayta en 
Collasuyo; Raurau y Chima en Cuntisuyo. Se encuentra también una coincidencia entre Anahuarque y el ceque del mismo nombre también ubicado en Cuntisuyo. Esto se debe a que probablemente estos ayllus fueron reducidos en San Jerónimo luego del proceso de consolidación del dominio español en el Cusco. Asimismo, de acuerdo con la crónica de Sarmiento de Gamboa (1988) estos ayllus corresponderían a los linajes de los Incas. Sin embargo, no es un objetivo del presente artículo estudiar la relación de los ayllus de San Jerónimo con los linajes reales, debido fundamentalmente a su antigüedad y complejidad.

El listado de ayllus de este centro poblado muestra el número de contribuyentes respec- tivo. Obsérvese que existe una diferenciación entre originarios y forasteros, siendo todos los originarios poseedores de tierras, mientras que entre los forasteros la mayoría de ellos no eran poseedores de estas. Ello plantea de inicio la doble definición del término Ayllu: por un lado, la existencia o no de un vínculo de parentesco (mediante la oposición originario-forastero) y por otro lado, la dimensión territorial de este, al especificarse la tenencia o no de tierras agrícolas. Ello fortalece el principio que sostiene que la base económica del ayllu es la actividad agrícola, por lo tanto, hay un fuerte vínculo territorial con los alrededores del centro poblado. Veamos ahora para ese mismo ańo el registro de predios rústicos:

Tabla 3

Predios rústicos de San Jerónimo

\begin{tabular}{|l|l|}
\hline 1836 & Propietario \\
\hline Patapaula & Convento de Santo Domingo \\
\hline Cayra & Convento de La Merced \\
\hline Concomarca & Don Juan Piroto \\
\hline Larapita & Don Juan Piroto \\
\hline Oscollo & Don Jorge Velasco \\
\hline Angostura & Doña Josefa Astete \\
\hline Buenavista & Doña Narsisa Aguirre \\
\hline Consevida & Don Gabino Jauregui \\
\hline Larapa-Grande & Beaterio de las Nazarenas \\
\hline Rurenhuasi & Don Blas Yanquiremachi \\
\hline Picopucyo & Don Dionicio Zeballos \\
\hline Tuñpampa & Don Ignacio Roca \\
\hline Limeñapampa & Don Blas Yanquirrimachi \\
\hline Munahuarmi & Doña Melchora Enriquez \\
\hline Ospa & Convento de Santo Domingo \\
\hline Percacalla & Don Santiago Fearo \\
\hline Inticancha & Don Bernabé Guamán \\
\hline Coyocacha & Don Manuel Zuta \\
\hline Guenchaquencha & Doña Agustina Orcohuaranca \\
\hline Chollo & Don Manuel Silvestre Gárate \\
\hline Pampachacra & Doña Isidora Angulo \\
\hline
\end{tabular}


Se observa la Tabla 3 elaborada a partir de las matriculas de predios rústicos del cercado que se encuentra en el Archivo Regional del Cusco (1836). Dicha tabla muestra que los predios rústicos tienen un propietario específico, mientras que en el ayllu prevalecía el vínculo colectivo, unido ya sea por un origen común a través del parentesco, o ya sea por un posible vínculo de proximidad en cuanto a la localización no solo de las tierras agrícolas, sino también del lugar de residencia, tal como se discutirá al final del presente artículo.

\section{EL DOCUMENTO DE 1876}

Tabla 4

Población urbana y rural en San Jerónimo en 1876

\begin{tabular}{|l|l|l|l|}
\hline Pueblos & Hombres & Mujeres & Total \\
San Jerónimo & 772 & 860 & 1632 \\
Total población urbana & 772 & 860 & 1632
\end{tabular}

\begin{tabular}{|l|l|l|l|}
\hline Caseríos P.R. & Hombres & Mujeres & Total \\
\hline Angostura y sus comprensiones (Hacienda) & 58 & 60 & 118 \\
\hline Buenavista (Hacienda) & 1 & 5 & 6 \\
\hline Cconchacalla & 34 & 30 & 64 \\
\hline Ccayra (Hacienda) & 57 & 41 & 98 \\
\hline Collollacta & 5 & 4 & 9 \\
\hline Cunca - marca (Hacienda) & 4 & 4 & 8 \\
\hline Huacapunco (Hacienda) & 3 & 5 & 8 \\
\hline Huacoto & 53 & 49 & 102 \\
\hline Huayllabamba (Hacienda) & 6 & 10 & 16 \\
\hline Huayrapunco (Hacienda) & 2 & 1 & 3 \\
\hline Larapa - chico (Hacienda) & 5 & 4 & 9 \\
\hline Larapa - grande (Hacienda) & 3 & 5 & 8 \\
\hline Limeñapata (Hacienda) & 1 & 1 & 2 \\
\hline Mamatunya (Hacienda) & 1 & 1 & 2 \\
\hline Manayhuarme y Quencoro (Hacienda) & 4 & 2 & 6 \\
\hline Miraflores de Tuño (Hacienda) & 9 & 13 & 22 \\
\hline Osccollo y Nascopampa (Haciendas) & 2 & 4 & 6 \\
\hline Parcohuaylla (Hacienda) & 6 & 7 & 13 \\
\hline Patapata (Hacienda) & 8 & 5 & 13 \\
\hline Pitupucllo e Inticancha (Haciendas) & 5 & 2 & 7 \\
\hline $\begin{array}{l}\text { Quichuaspata, Lloquemaco y Fiero - ccata } \\
\text { (Haciendas) }\end{array}$ & 25 & 26 & 51 \\
\hline Rau - rau & 33 & 37 & 70 \\
\hline Sayllanahuarque & 163 & 179 & 342 \\
\hline Suncco & 36 & 31 & 67 \\
\hline Total población rural & 524 & 526 & 1050 \\
\hline Población total del distrito & 1296 & 1386 & 2682 \\
\hline
\end{tabular}


La Tabla 4 ha sido extraída del Censo realizado en Cusco en 1876 (Archivo General de la Nación, Censo de la República del Perú 1876). Se observa aquí por primera vez una diferenciación entre población urbana y rural. La población urbana podría interpretarse como aquella que residía en el centro poblado de San Jerónimo, (1632 habitantes), mientras que la población rural la conformaban las personas que vivían en los caseríos (1050 habitantes). Uno de los elementos más interesantes de este cuadro es el hecho que dentro del área rural se hace una distinción entre caseríos considerados como haciendas y caseríos sin esta denominación, y son los primeros, la mayoría.

Si comparamos esta Tabla con la 3, que corresponde a los predios rústicos, muchos de ellos son considerados aquí como haciendas. Sin embargo, no se consideraban como tales Cconchacalla, Collollacta, Huacoto, Rau-rau, Sayllanahuarque y Suncco. De estos últimos, Cconchacalla no ha podido ser ubicado por el momento; Collollacta aparece en el mapa del Instituto Geográfico Nacional (1973) como una hacienda; Huacoto es un centro poblado, ubicado en la parte alta del valle, al Noreste de San Jerónimo, que aparece también en el mapa del IGN antes citado; Rau-rau aparece en la lista de ayllus de la Citua y en el de las matrículas de indígenas de 1836; Sayllanahuarque podría corresponder al centro poblado de Saylla, ubicado aproximadamente, $6 \mathrm{Km}$. al sureste de San Jerónimo. En tal sentido, no se ha podido comprobar aún si el ayllu Anahuarque, que aparece en la lista de 1836, tiene alguna relación con el centro poblado de Saylla. Finalmente, Suncco es un lugar que no ha podido ser identificado aún. Lo interesante de este cuadro es que encontramos ya una diferenciación entre el concepto de urbano y rural, hecho que no se observa en los libros de matrículas de predios, en los cuales el término rural parece asociarse al término rústico. Para el caso de Rau-rau, podríamos estar en condiciones de formular la hipótesis que se trata del ayllu que aparece en la relación de 1836. De ser así, podría decirse que dicho ayllu estaba localizado fuera del centro poblado mismo. Sin embargo, esto lo discutiremos más adelante.

\section{San Jerónimo en 1888}

El documento base utilizado para realizar la Tabla 5 es el Libro de Matrícula de contribuyentes de la parroquia de San Jerónimo Legajo 17, Libro 24, el mismo que se encuentra en el Archivo Regional del Cusco (1888).

Tabla 5

Contribución personal en San Jerónimo - Año 1888

\begin{tabular}{|c|c|}
\hline Contribución Personal & \\
\hline No especificado & 97 \\
\hline Collana & 36 \\
\hline Anahuarqque & 95 \\
\hline Quishuarpata & 2 \\
\hline Chahuanccoscco & 29 \\
\hline Ccallampata & 11 \\
\hline Sucso & 42 \\
\hline Aucaille & 37 \\
\hline Accamana & 29 \\
\hline Huaccoto & 21 \\
\hline Roccaquirao & 22 \\
\hline Apumaita & 16 \\
\hline Raurau & 22 \\
\hline Chima & 22 \\
\hline Sorama & 24 \\
\hline Ccollanccas & 11 \\
\hline Andamachay & 8 \\
\hline Ccaira & 20 \\
\hline Larapita & 2 \\
\hline Fienocata & 6 \\
\hline Chillicpampa & 3 \\
\hline Larapa & 8 \\
\hline Huaillabamba & 5 \\
\hline Munaihuarme & 1 \\
\hline Angostura & 10 \\
\hline & 579 \\
\hline
\end{tabular}


La Tabla 5 es un resumen del listado de contribuyentes correspondiente al año respectivo, mostrándose aquí únicamente el número de contribuyentes por cada grupo. Uno de los rasgos más importantes de este documento es que aquí ya no se hace diferencia entre indígenas, gremios, castas, y eclesiásticos tal como ocurría durante el período comprendido entre 1826 y 1851 . Asimismo, se observa que no se hace distinción entre ayllu, hacienda o caserío, hecho que dificulta la comprensión de este registro. Sin embargo, resulta interesante encontrar los ayllus que aparecían en el documento de 1836 y que no figuran en el censo de 1876.
El problema por resolver es determinar si estos nombres expresan la continuidad de los ayllus o si se trata de unidades territoriales que fueron utilizadas para poder realizar el registro de los contribuyentes. La información contenida en este documento sugiere entonces diversas hipótesis que permitirían precisar el carácter de los ayllus de San Jerónimo, pero que se mantienen difusos en cuanto a espacialidad.

Estableciendo una correspondencia entre la relación de ayllus de 1836 y el registro de contribución personal de 1888 podemos plantear la tabla siguiente: (Tabla 6):

\section{Tabla 6}

Cuadro comparativo de ayllus en 1836 con el registro de 1888

\begin{tabular}{|l|l|l|}
\hline $\begin{array}{l}\text { Contribución Personal en } \\
1888\end{array}$ & Ayllus en 1836 & Categorías \\
\hline$\ldots . .$. & - & No determinado \\
\hline Collana & Ccollana & Ayllu \\
\hline Anahuarqque & Anahuarqui & Ayllu \\
\hline Quishuarpata & - & Hacienda (1876) \\
\hline Chahuanccoscco & Chahuanccoscco & Ayllu \\
\hline Ccallampata & Ccallampa & Ayllu \\
\hline Sucso* & - & Ayllu en 1768 \\
\hline Aucaille* & - & Ayllu en 1768 \\
\hline Accamana & Uruhuaccama & Ayllu \\
\hline Huaccoto & - & Caserío (1876) \\
\hline & Huiccahuiray & Ayllu \\
Roccaquirao & Guiccahuiray) & \\
\hline Apumaita & Apumayta & Ayllu \\
\hline Raurau & Raurau & Ayllu \\
\hline Chima & Chima & Ayllu \\
\hline Sorama & Sorama & Ayllu \\
\hline Ccollanccas & Ccollanas & Ayllu \\
\hline Andamachay & Andamachay & Ayllu \\
\hline Ccaira & - & Hacienda (1836 y 1876) \\
\hline Larapita & - & Hacienda (1836 y 1876) \\
\hline Fienocata & - & No determinado \\
\hline Chillicpampa & - & No determinado \\
\hline Larapa & - & Hacienda (1836 y 1876) \\
\hline
\end{tabular}




\begin{tabular}{|l|l|l|}
\hline Huaillabamba & - & Hacienda (1876) \\
\hline Munaihuarme & - & Hacienda (1836 y 1876) \\
\hline Angostura & & Hacienda (1836 y 1876) \\
\hline No figura & Orccon & Ayllu \\
\hline
\end{tabular}

Haciendo esta comparación, no figuran aquí todas las haciendas que pertenecían a San Jerónimo. Ello no significa que estas hayan desaparecido, sino que aparecen en otro rubro (correspondiente al ramo de predios rústicos e industriales) que no mostramos aquí. Sin embargo, resulta de mucho interés observar que dos ayllus que no figuran en el documento de 1836 sí aparecen aquí. Se trata de los ayllus Sucso y Aucaille, los cuales fueron históricamente considerados como ayllus de origen Inca, tal como lo vimos en relación con las direcciones de la $\mathrm{Ci}$ tua y los Ceques, en los que aparecían los ayllus Sucsu y Aucailli como pertenecientes al Antisuyo. Asimismo, según Sarmiento de Gamboa estaban asociados a los incas Yahuar Huácac (Aucailli) y Viracocha (Sucsu). Probablemente, estos ayllus no aparecieron en el documento de 1836 debido a que por tener esta categoría estaban exentos del pago de las contribuciones.

Efectivamente, tal como lo sostiene David Garret (2009), basándose en un documento que se encuentran en el Archivo Regional del Cusco [Corregimiento, Administrativo, Legajo 94 (1767-84)] en 1768 se registraban en San Jerónimo los ayllus Sucso de Nobles y Aucailli de Nobles. Volviendo a la lectura de la Tabla 6, a pesar de que no se establece una diferenciación entre ayllu, hacienda o predio rústico, aparecen los nombres de todos los ayllus que aparecían en 1836, incluyendo estos dos últimos, los cuales habían figurado en 1768. El problema es aquel que fue enunciado al inicio del artículo: ¿Cuál fue el criterio para realizar este registro? ¿Fue solo en términos de parentesco? Si fue un criterio de orden territorial - que parece ser lógico por el hecho deque las haciendas estaban consideradas en 1876 como parte del ámbito rural - falta aclarar cuál fue el criterio empleado para los ayllus.

\section{Reconstrucción cartográfica de San Jerónimo y alrededores}

A continuación, presentamos unos mapas que nos permiten tener una idea de la organización espacial y territorial de San Jerónimo, la misma que, al conjugarse con los datos de archivo antes expuestos nos permitirán formular las bases para establecer las conclusiones del presente artículo. 


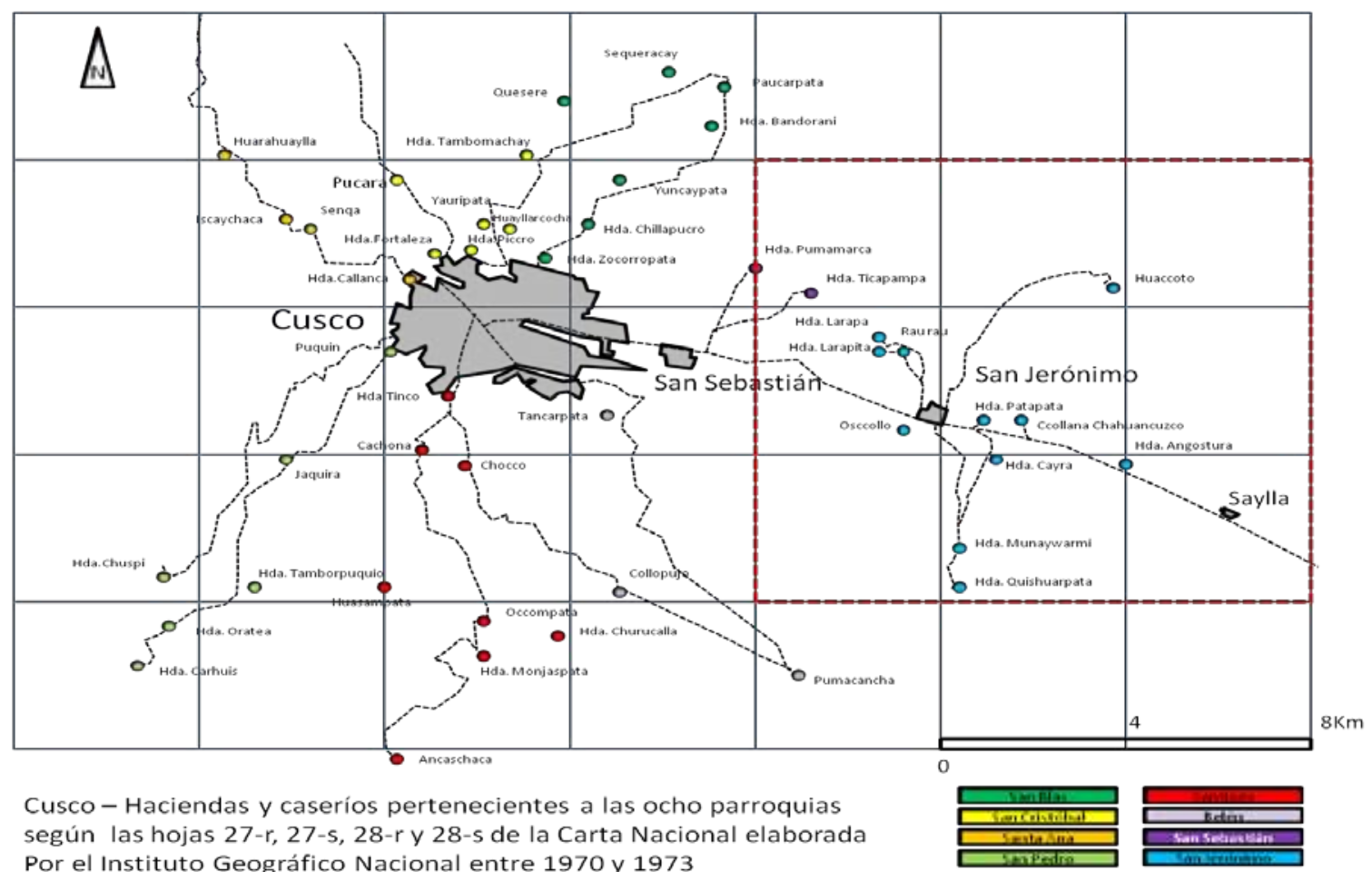

Figura 3. Haciendas y caseríos pertenecientes a las ocho parroquias del Cusco entre 1970 y 1973

La Figura 3 muestra la ubicación de San Jerónimo con respecto a la ciudad del Cusco entre 1970 y 1973, y fue elaborado a partir de la interpretación de las hojas 27-r, 27-s, 28-r y 28-s de la Carta Nacional escala 1:100000 del Instituto Geográfico Nacional. No figuran aquí todas las haciendas pertenecientes a las parroquias del Cusco que existían durante el siglo XIX, pero sí aquellas que habían sido registradas durante los primeros años de la década de los setenta en los mencionados mapas. La ubicación de las haciendas se ha logrado a partir de la identificación sobre el mapa de algunas haciendas que aparecen en el Libro de Matrículas del Cercado (1888).

Obsérvese que las haciendas tenían una localización específica, a manera de extensión de sus respectivos barrios urbanos. En esta distribución territorial, los caminos rurales deben haber tenido una influencia fundamental en la organización de las parroquias, pues la sede de las parroquias de San Pedro, San Cristóbal, San Blas, Santa Ana, Santiago y Belén estaban integradas al área urbana, vale decir a la parroquia Matriz, mientras que las parroquias de San Sebastián y San Jerónimo estaban más alejadas, hecho que contrasta con la Figura 1, donde se observa que San Sebastián está integrada al casco urbano actual, mientras que San Jerónimo está próximo a serlo. Cabe advertir que muchas haciendas de los libros de matrículas de 1888 no aparecen, en este mapa, debido a muchas razones, por un lado, su aparente desaparición, por otro lado, el proceso de urbanización durante el siglo XX, sobre todo en la parroquia de San Blas. Para el caso de San Jerónimo, la Figura 3 muestra solo algunas haciendas, por razones de escala; sin embargo, en la Figura 4 tendremos una representación más completa. 


\section{SAN JERÓNIMO - Haciendas y caseríos}

\section{según la hoja 28-s Cuzco levantada en 1973}

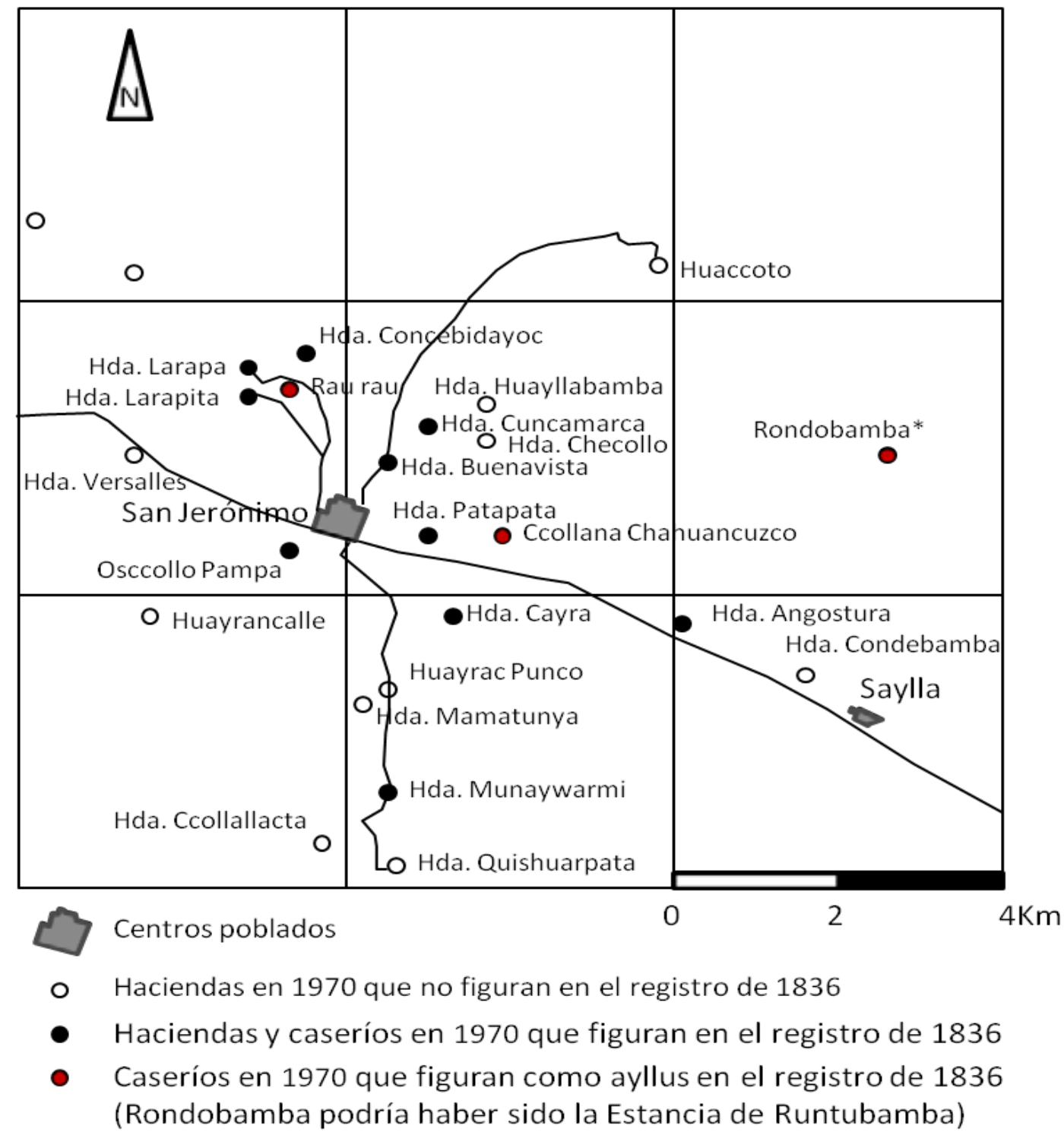

Figura 4. Haciendas y caseríos de San Jerónimo en 1973

El mapa correspondiente a la Figura 4 ha sido elaborado a partir de la Hoja 28-r Cuzco de la Carta Nacional escala 1/100000 del IGN. Muestra, con mayor detalle, las estancias y caseríos de los alrededores de San Jerónimo en 1973. Puede observarse que varios de estos caseríos y haciendas existían ya en 1836. Otros de estos caseríos aparecen en los registros de 1876 y 1888 , tal es el caso de Huaccoto. Y lo que es notable, a su vez, es la presencia de Rau Rau y Ccollana Chahuancuzco, que en el registro de 1836 aparecen como los ayllus Raurau, Ccollana y Chahuanccoscco, respectivamente. Esta información permite plantear la hipótesis que los ayllus tuvieron extensiones territoriales en función de la proximidad de sus áreas agrícolas. La estancia de Runtubamba, probablemente, es el caserío llamado Rondobamba. Finalmente, lugares como Raurau 


\section{San Jerónimo}

\section{Manzanas y calles en 2016}

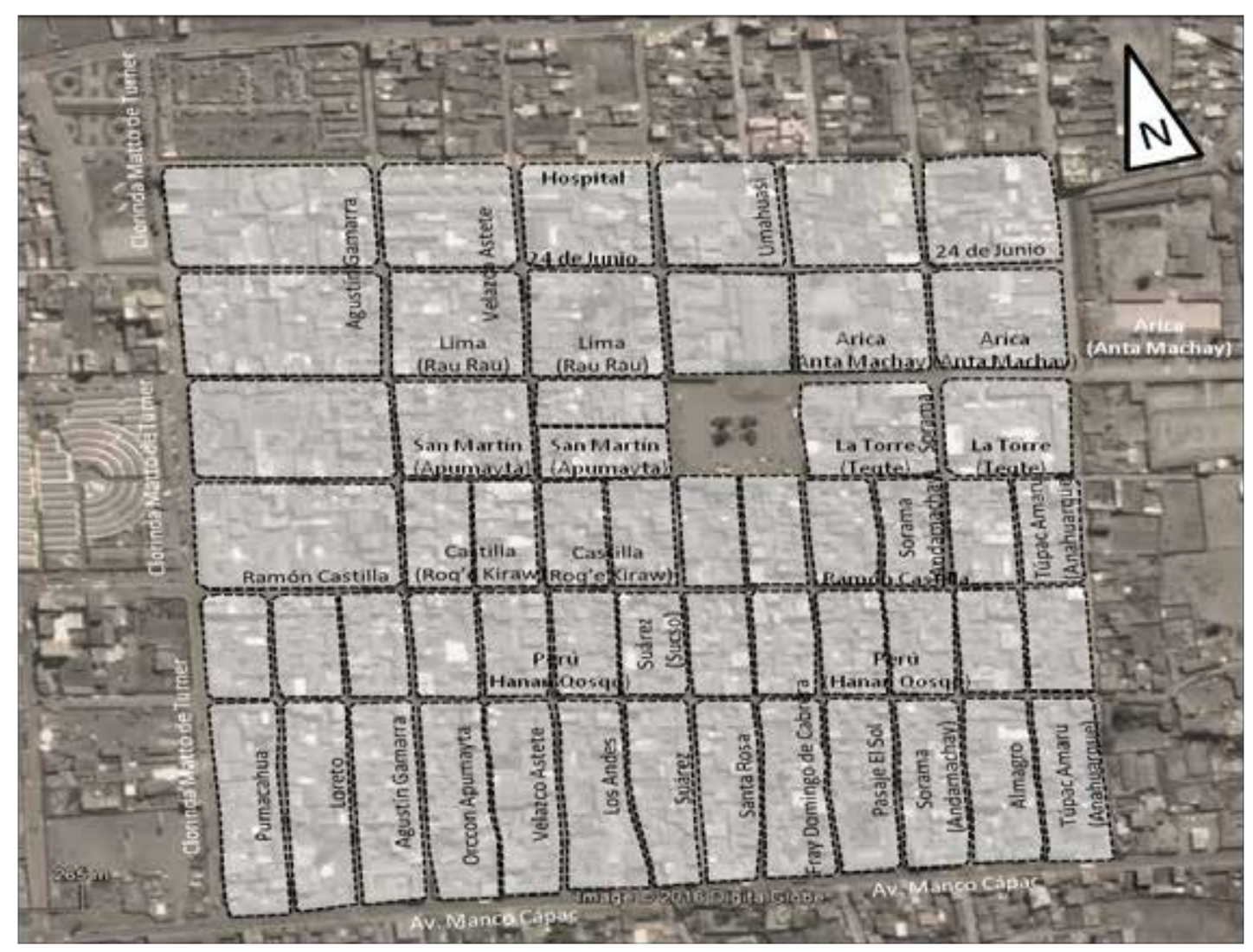

132.5

$265 \mathrm{~m}$

$\mathrm{O}$

Plano elaborado a partir de Google Earth y Las imágenes de Street Wiew.

Figura 5. Manzanas y calles de San Jerónimo en 2016

y Ccollana Chahuancuzco aparecen también en los registros de 1888, sin embargo, este último no aparece en el censo de 1876. Queda aún por investigar si el ayllu Anahuarque estuvo ubicado en el centro poblado de Saylla.

La figura 5 muestra el trazado actual de San Jerónimo, donde se puede apreciar la estructura de manzanas propia de un pueblo de reducción, y donde aparecen los nombres de las calles del mismo. Lo interesante de esto es que en muchas calles aparece no solo el nombre actual de las mismas, sino también el nombre que tenían antiguamente, nombres que en la mayoría de los casos coinciden con aquellos de los antiguos ayllus de San Jerónimo, Tabla 7:
Tabla 7

Comparación entre los nombres actuales y los nombres antiguos de algunas calles de San Jerónimo.

\begin{tabular}{|l|l|}
\hline Nombre actual & Nombre original \\
\hline Túpac Amaru & Anahuarque \\
\hline Sorama & Andamachay \\
\hline Arica & Antamachay \\
\hline San Martín & Apumayta \\
\hline $\begin{array}{l}\text { Orccon } \\
\text { Apumayta }\end{array}$ & $\begin{array}{l}\text { Orccon } \\
\text { Apumayta }\end{array}$ \\
\hline Lima & Rau Rau \\
\hline Castilla & Roq'e Kiraw \\
\hline Suárez & Sucso \\
\hline La Torre & Teqte \\
\hline
\end{tabular}


Puede verse que no aparecen todos los nombres de los ayllus, como es el caso de Acamana y Aucaille, así como también Chahuanccoscco. Sin embargo, esta información permitiría establecer finalmente las hipótesis acerca de la dimensión territorial del concepto de ayllu. Tom Zuidema, en su libro sobre los ceques del Cusco (1998) [1964] presenta un croquis de San Jerónimo donde aparecen las calles de este con los nombres de los ayllus. Es probable que Zuidema haya recorrido San Jerónimo, en un momento, en el que los nombres originales de las calles aún no se habían modificado por los actuales. Coinciden aquí las ubicaciones de Anahuarque, Surama, Sucsu, Raurau y Andamachay; sin embargo, no aparecen Teqte ni Roq'e Kiraw. Es interesante notar que Zuidema identifica las calles Acamana y Aucaylli, las mismas que no aparecen en la señalización actual. Asimismo, indica que sobre el actual Fray Domingo de Cabrera la calle se llamaba Collana. Sin embargo, es necesario hacer un trabajo de archivo más importante a fin de tener la certeza que estas calles hayan sido el lugar de residencia de los integrantes de los ayllus allí nombrados.

\section{Conclusiones}

Como se dijo, en la introducción del presente artículo, no se pretende aquí resolver el problema planteado inicialmente ni formular todas las hipótesis necesarias para lograr resolverlo; sin embargo, la revisión de los da- tos correspondientes a los tres momentos del siglo XIX analizados y su comparación entre sí y con respecto a la cartografía correspondiente a los siglos XX y XXI permite afirmar que los ayllus de San Jerónimo tuvieron una ubicación específica, tanto en el ámbito urbano como en el área rural circundante.

Los casos de Raurau y Chahuanccoscco muestran que estos se extendían como caseríos en los alrededores de San Jerónimo, y específicamente, Raurau como un ayllu que tenía un área rural y probablemente una ubicación urbana a lo largo de la actual calle Lima de San Jerónimo. Esta afirmación se ve fortalecida por el hecho de que la extensión de esta calle da inicio al camino que conduce al caserío Raurau. El problema de fondo es que las demás calles con los nombres de los ayllus no dejan claro si sus extensiones viales conducían, necesariamente, a las tierras agrícolas pertenecientes a estos. Pero lo importante de esta comparación entre ayllus, haciendas y estructura urbana y rural es que el concepto de ayllu no fue únicamente un tema de linaje o descendencia, sino que también tuvo una connotación espacial, la misma que puede hacerse más sólida en la medida en que se puedan hallar más datos que permitan precisar la localización de las tierras agrícolas y los lugares de residencia de los habitantes de dichos ayllus. Por tal motivo, como hipótesis fundamental podríamos decir que mientras que las tierras agrícolas de los ayllus de San Jerónimo estarían ubicadas en sus alrededores, los integrantes de estos podrían haber tenido su lugar de residencia en el área urbana en función a los nombres de las calles de este. 


\section{Referencias}

Basadre, J. (2005). Historia de la República del Perú [1822-1933] (Tomo I). Lima, Perú: Edición Orbis Ventures S.A.C.

De Molina, C. (2008). Relación de las Fábulas y Ritos de los Incas. Lima, Perú: Fondo Editorial de la Universidad San Martín de Porres.

Dirección General de estadística. (1878). Censo de la República del Perú, formado en 1876 (Tomo 4). Lima, Perú: Imprenta del Teatro.

Garret, D. (2009). Sombras del Imperio. La Nobleza indigena del Cuzco, 17501825. Lima, Perú, Instituto de Estudios Peruanos IEP.

Instituto Geográfico Nacional (1970-1973). Carta Nacional Escala 1: 100,000. Hojas 27-r (Urubamba); 27-s (Calca); 28-r (Tambobamba); 28-s (Cuzco).

Rowe, J. H. (diciembre, 1981). Una relación de los adoratorios del antiguo Cuzco. Histórica, 5(2), 209-261.

Zuidema, T. (1995). El sistema de Ceques del Cuzco. La organización social de la capital de los Incas. Perú: Fondo Editorial de la Pontificia Universidad Católica del Perú.

Archivo General de la Nación (1839). Libro de Matrícula de Patentes del Cusco co- rrespondiente al año 1839. Sección de Contribuciones (H-4-1976). Archivo General de la Nación, Lima, Perú.

Archivo General de la Nación. (1826). Libro padrón de patentes, predios rústicos y urbanos del Cuzco, asi como del cabildo eclesiástico. Año: 1826. Sección de Contribuciones (H-4-1614). Archivo General de la Nación, Lima, Perú.

Archivo Regional del Cusco. Tesorería Fiscal. Año 1836. Libro extracto de predios rústicos del Cuzco. Legajo 16 (Libro 2). Archivo Regional del Cusco, Perú.

Archivo Regional del Cusco. Tesorería Fiscal. Año 1836. Libro extracto de matrícula de indigenas del Cuzco. Legajo 16 (Libro 1). Archivo Regional del Cusco, Perú.

Archivo Regional del Cusco. Año 1888. Tesorería Fiscal. Matricula de contribuyentes de la provincia del cercado. Legajo 17 (Libro 17 Parroquia de San Pedro; Libro 18 Parroquia de San Cristóbal; Libro 19 Parroquia de San Blas; Libro 20 Parroquia de Santa Ana; Libro 21 Parroquia Matriz; Libro 22 Parroquia de Santiago; Libro 23 Parroquia de San Sebastián; Libro 24 Parroquia de San Jerónimo, Libro 25 Parroquia de Belén 
